\title{
Atomically-thin population of colloidal CdSe nanoplatelets: growth of rolled-up nanosheets and strong circular dichroism induced by ligand exchange
}

Daria A. Kurtina ${ }^{\dagger}$, Alexey V. Garshev ${ }^{\dagger,}$, Irina S. Vasil'eva ${ }^{\S}$, Vladimir V. Shubin ${ }^{\S}$, Alexander M. Gaskov $^{\dagger}$, and Roman B. Vasiliev ${ }^{\dagger, \dot{t}^{*}}$

${ }^{\dagger}$ Department of Chemistry, Lomonosov Moscow State University, 119991, Moscow, Russia

${ }^{7}$ Department of Materials Science, Lomonosov Moscow State University, 119991, Moscow, Russia

${ }^{\S}$ Bach Institute of Biochemistry, Research Center of Biotechnology of the Russian Academy of Sciences, Leninsky Ave. 33, Bld. 2, 119071 Moscow, Russia

*E-mail: romvas@inorg.chem.msu.ru.

\section{Supporting Information}

1. Synthetic details for thicker CdSe nanosheets (CdSe463 and CdSe463/CdS samples).

2. FTIR analysis of ligand composition of CdSe3940A, CdSe394TGA and CdSe394AcCys samples.

3. Additional TEM images of CdSe3940A samples.

4. Additional TEM images of CdSe394TGA and CdSe394AcCys samples.

5. Results of elemental composition analysis from STEM-EDX mapping for CdSe394AcCys sample.

6. Additional absorption and photoluminescence spectra for CdSe394OA samples.

7. Analysis of CdSe463/CdSAcCys samples with different lateral sizes. 


\section{Synthetic details for thicker CdSe nanosheets (CdSe463 and CdSe463/CdS samples)}

The CdSe463 nanosheets (CdSe463OA NSs sample) were synthesized using the adapted method from [1]. Growth of $\mathrm{CdS}$ shell on both basal planes of $\mathrm{CdSe} 463$ nanosheets (CdSe463/CdSOA sample) was carried out using the protocol of [2]. The following chemicals were purchased from Sigma-Aldrich and used without additional purification: cadmium acetate dihydrate (Cd(OAc)2·2H2O, $\geq 98 \%$ ), selenium powder (Se), (99,99\%), 1-octadecene (ODE) (90\%), trioctylphosphine (TOP) (95\%), oleic acid (OA) (95\%), n-methylformamide (NMF) $(99 \%$ ), sodium sulfide nonahydrate (Na2S·9H2O, 99,9\%), N-acetyl-L-cysteine (AcCys, $\geq 99 \%$ ), and solvents.

\section{Synthesis of CdSe463 nanosheets.}

Briefly, a mixture containing $0.13 \mathrm{~g}$ of cadmium acetate dihydrate, $0.08 \mathrm{ml}$ of oleic acid, and 10 $\mathrm{ml}$ of degassed octadecene was heated to $230^{\circ} \mathrm{C}$ under argon flow. After that, $100 \mu \mathrm{l}$ of $1 \mathrm{M}$ solution of selenium in trioctylphosphine was injected and the growth of the NSs was continued for $30 \mathrm{~min}$. Then $1 \mathrm{ml}$ of oleic acid was injected and the reaction mixture was cooled down to room temperature. Nanosheets were precipitated by equal volume of acetone and separated by centrifugation at $6000 \mathrm{rpm}$. After 2-3 cycles of precipitation and redispersion, the solutions of the NSs in hexane with minimum impurity content were obtained.

Synthesis of CdSe463 nanosheets with extended lateral size was performed similarly to CdSe394OA samples by successive iteration of seed growth as described in the main text.

Synthesis of CdSe463/CdS nanosheets.

Briefly, $1 \mathrm{ml}$ of hexane solution of CdSe463 NSs was placed in 4 ML vial and $1 \mathrm{ML}$ of a $0.1 \mathrm{M}$ $\mathrm{Na} 2 \mathrm{~S}$ solution in NMF was added. After this, the color of NSs changed on red, and transfer of NSs from nonpolar hexane to NMF polar phase was starteded. The polar phase was rinsed several times with hexane. Then, the sulfide-covered NSs were were precipitated by equal volume of toluene and separated by centrifugation at $6000 \mathrm{rpm}$. Afterward, $1 \mathrm{ML}$ of a 0,2 M solution of cadmium acetate in NMF was added to the precipitate and left for 40 min to grow a CdS shell. The resulting NSs were precipitated using an equal volume of acetonitrile and toluene, separated by centrifugation at $6000 \mathrm{rpm}$, and washed by precipitation and redispersion, as described above to remove unreacted precursors. The resulting nanosheets were dispersed in hexane/oleic acid mixture to cover their surface by oleic acid and purified by 2-3 cycles of precipitation and redispersion with hexane/acetone as solvent/ antisolvent. Final sample was obtained in hexane.

Synthesis of CdSe463AcCys and CdSe463/CdSAcCys nanosheets.

Ligand exchange of oleic acid to $\mathrm{N}$-acetyl-L-cysteine was performed similary to CdSe394OA sample as described in main text.

References.

[1]. Shlenskaya, N. N.; Yao, Y.; Mano, T.; Kuroda, T.; Garshev, A.V.; Kozlovskii, V.F.; Gaskov, A.M.; Vasiliev, R.B.; Sakoda, K. Scroll-like alloyed $\mathrm{CdS}_{\mathrm{x}} \mathrm{Se}_{1-\mathrm{x}}$ nanoplatelets: facile synthesis and detailed analysis of tunable optical properties. Chemistry of Materials. 2017, 29, 579-586.

[2]. Saidzhonov, B.M.; Kozlovsky, V.F.; Zaytsev, V.B.; Vasiliev, R.B. Ultrathin CdSe/CdS and $\mathrm{CdSe} / \mathrm{ZnS}$ core-shell nanoplatelets: The impact of the shell material on the structure and optical properties. Journal of Luminescence, 2019, 209, 170-178. 
2. FTIR analysis of ligand composition of CdSe3940A, CdSe394TGA and CdSe394AcCys samples.

(a)

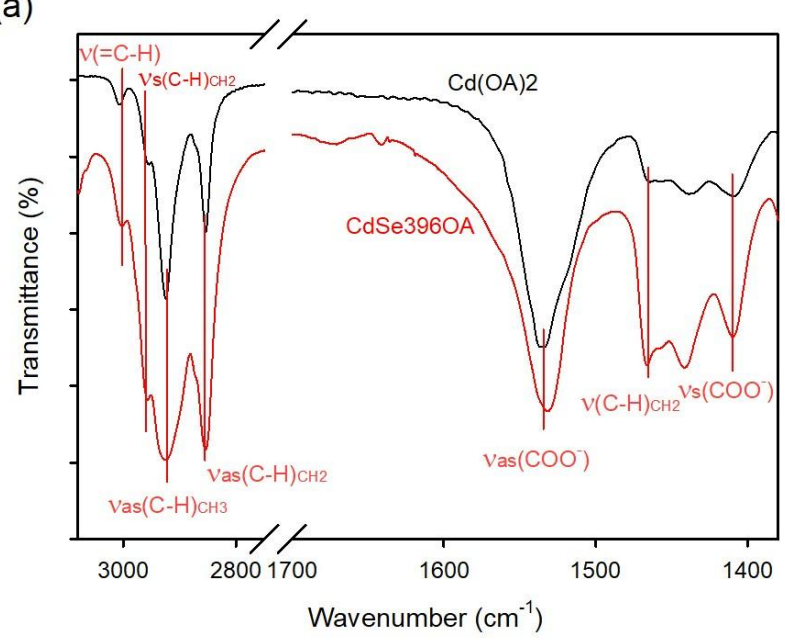

(b)

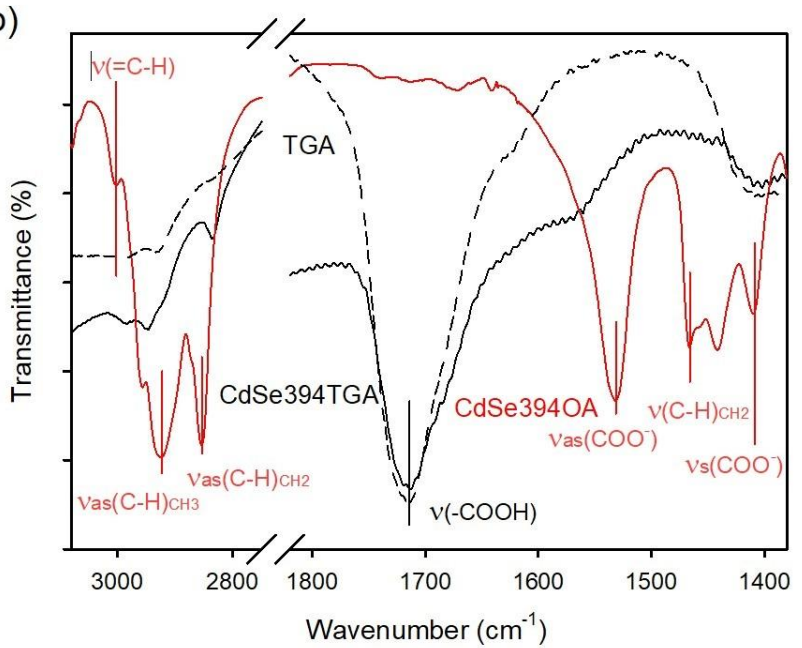

(c)

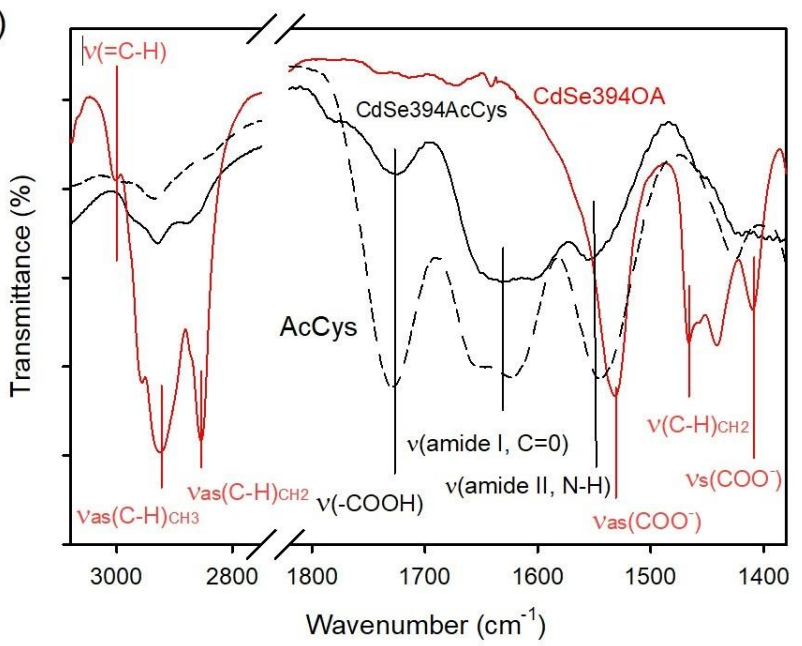

Figure S1. (a) FTIR spectrum of CdSe394OA nanosheets. Red solid line - OA capped sample, black solid line - cadmium oleate $\mathrm{Cd}(\mathrm{OA})_{2}$ reference. $(b, c)$ FTIR spectra of CdSe394OA NSs before and after ligand exchange with TGA $(b)$ and AcCys (c) ligands. Red solid line - initial OA capped sample, black solid line - TGA or AcCys exchanged samples, black dotted line - pristine TGA or AcCys references. Positions of the main vibration bans are marked. The spectra are offset for clarity. 
3. Additional TEM images of CdSe3940A samples.

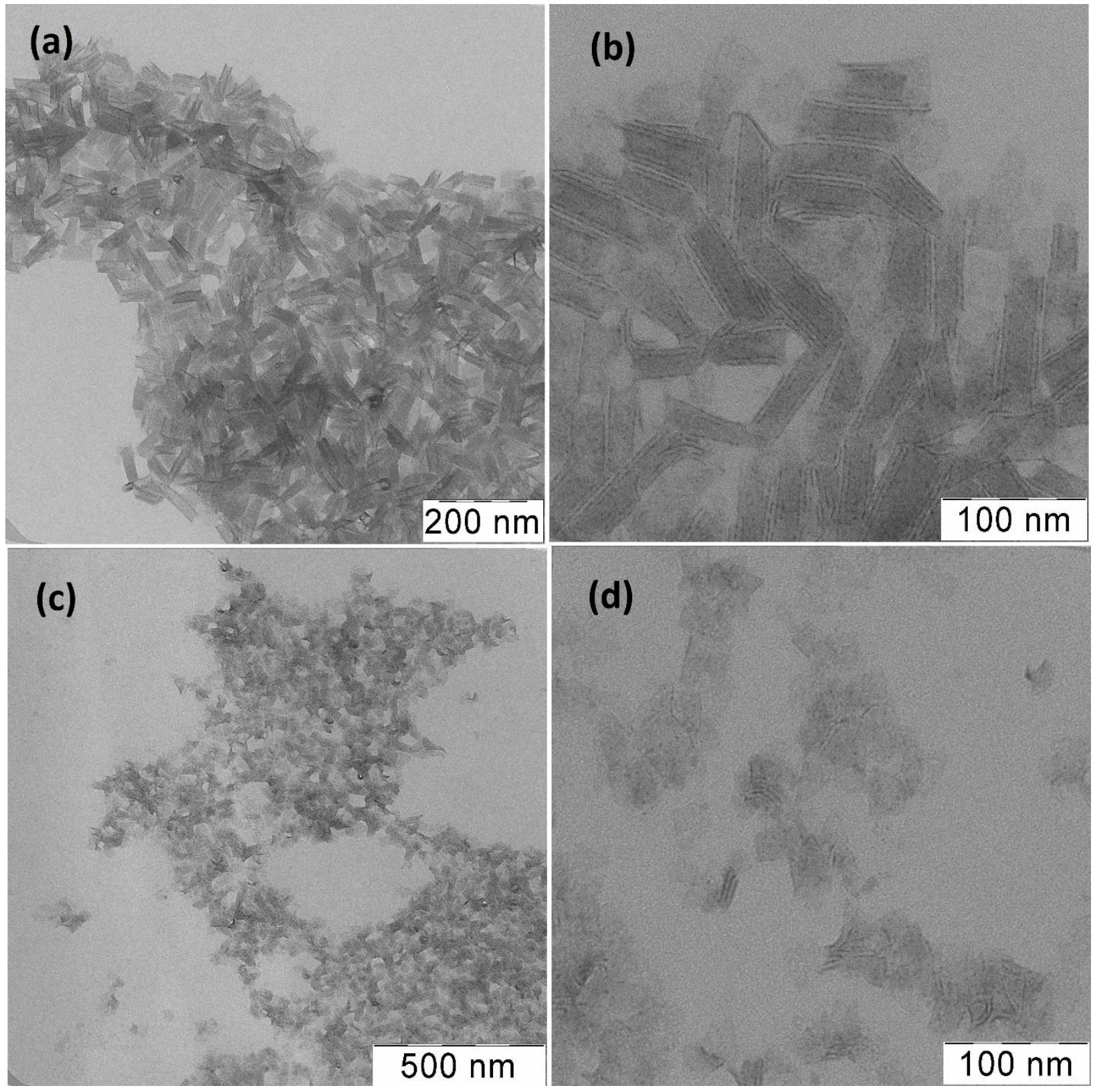

Figure S2. Overview low-resolution TEM images of CdSe394OA NS samples grown at $160^{\circ} \mathrm{C}(a$, b) and $120^{\circ} \mathrm{C}(c, d)$ temperature of growth. 
4. Additional TEM images of CdSe394TGA and CdSe394AcCys samples.

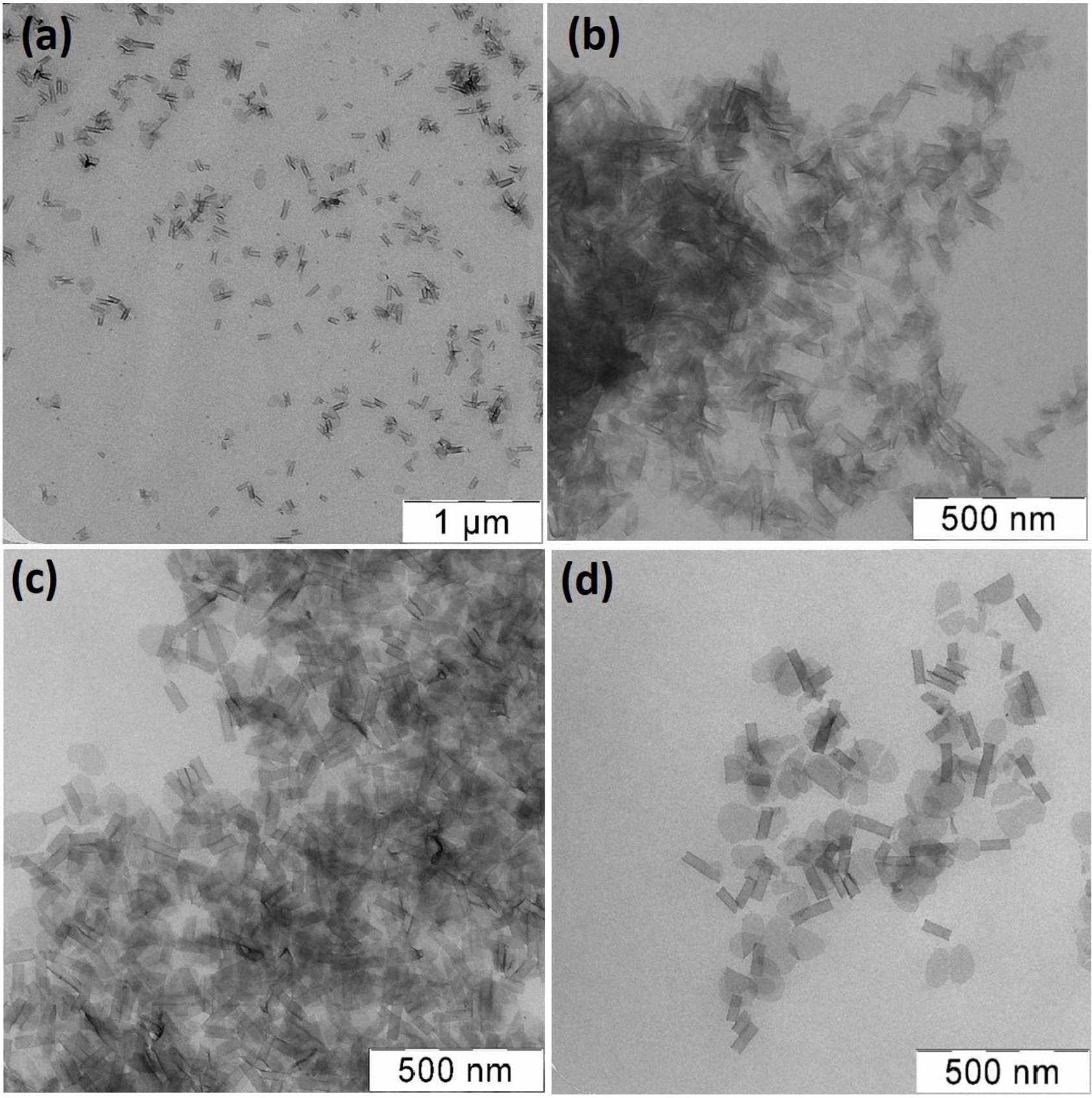

Figure S3. Overview low-resolution TEM images of the same CdSe394OA NS sample exchanged with TGA $(a, b)$ and AcCys $(c, d)$ ligands. 
5. Results of elemental composition analysis from STEM-EDX mapping for CdSe394AcCys sample
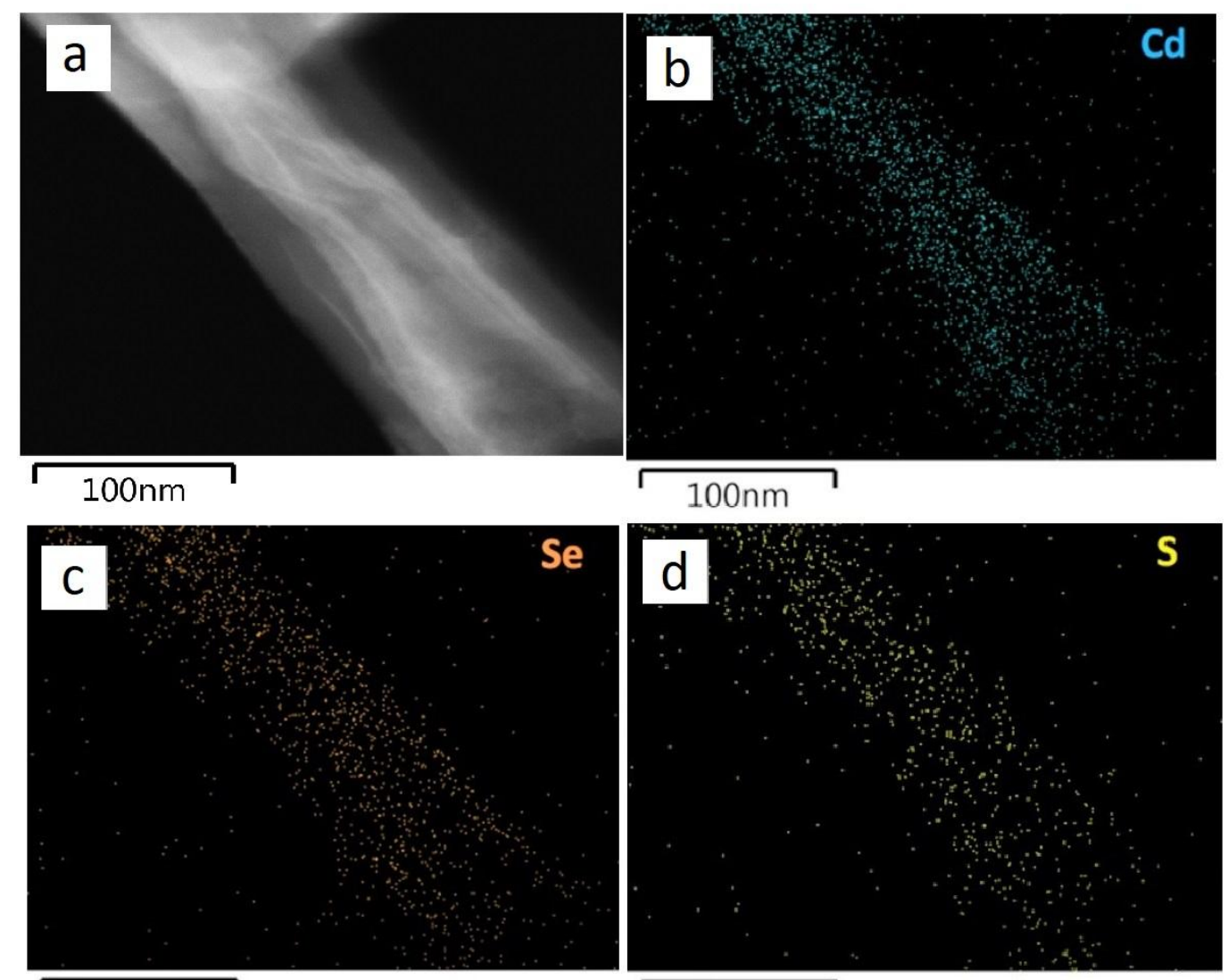

$100 \mathrm{~nm}$

$100 \mathrm{~nm}$
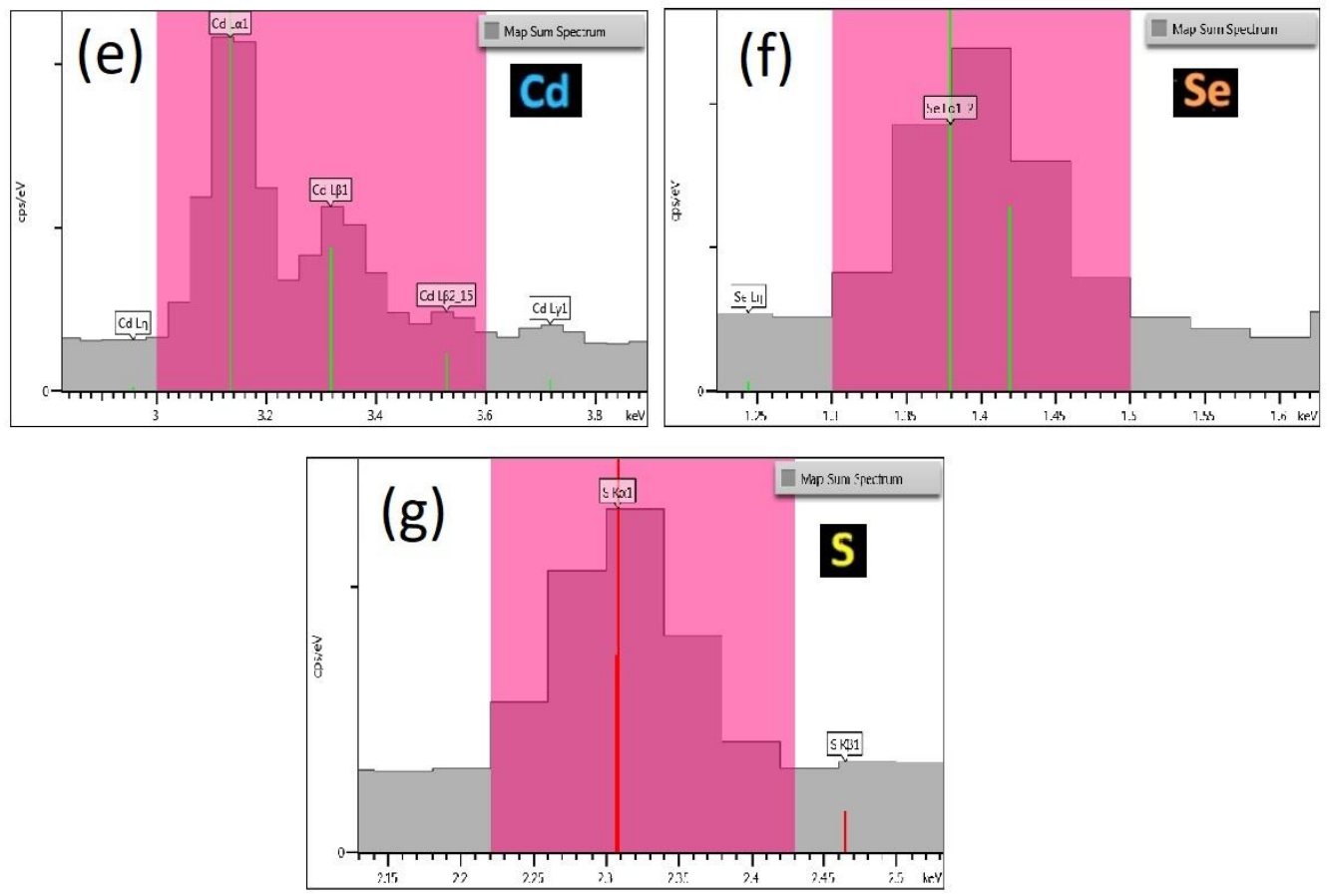

Figure S4. (a-d) Additional images of STEM-EDX mapping from the single CdSe394AcCys nanoscroll, where $C d$, Se, and S are colored by blue, orange, and yellow, respectively. $(e-g)$ Integrated EDX spectra collected from the analyzed area. 
Table S1. Elemental composition data from integrated STEM-EDX analysis collected on various areas of CdSe394AcCys nanosheets.

\begin{tabular}{|c|c|c|c|}
\hline Spectrum Label & Cd, at.\% & Se, at.\% & S, at.\% \\
\hline Map \#1 & 41.36 & 29.43 & 29.21 \\
\hline Map \#2 & 41.94 & 30.19 & 26.87 \\
\hline Map \#3 & 42.59 & 29.71 & 27.70 \\
\hline Map \#4 & 42.45 & 31.57 & 25.98 \\
\hline Map \#5 & 41.91 & 31.46 & 26.63 \\
\hline
\end{tabular}

Average atomic compositions are [Cd] -42.05 at.\%, [Se] - 30.47 at.\%, and [S] - 27.48, at.\%. 


\section{Additional absorption and photoluminescence spectra of CdSe3940A samples}

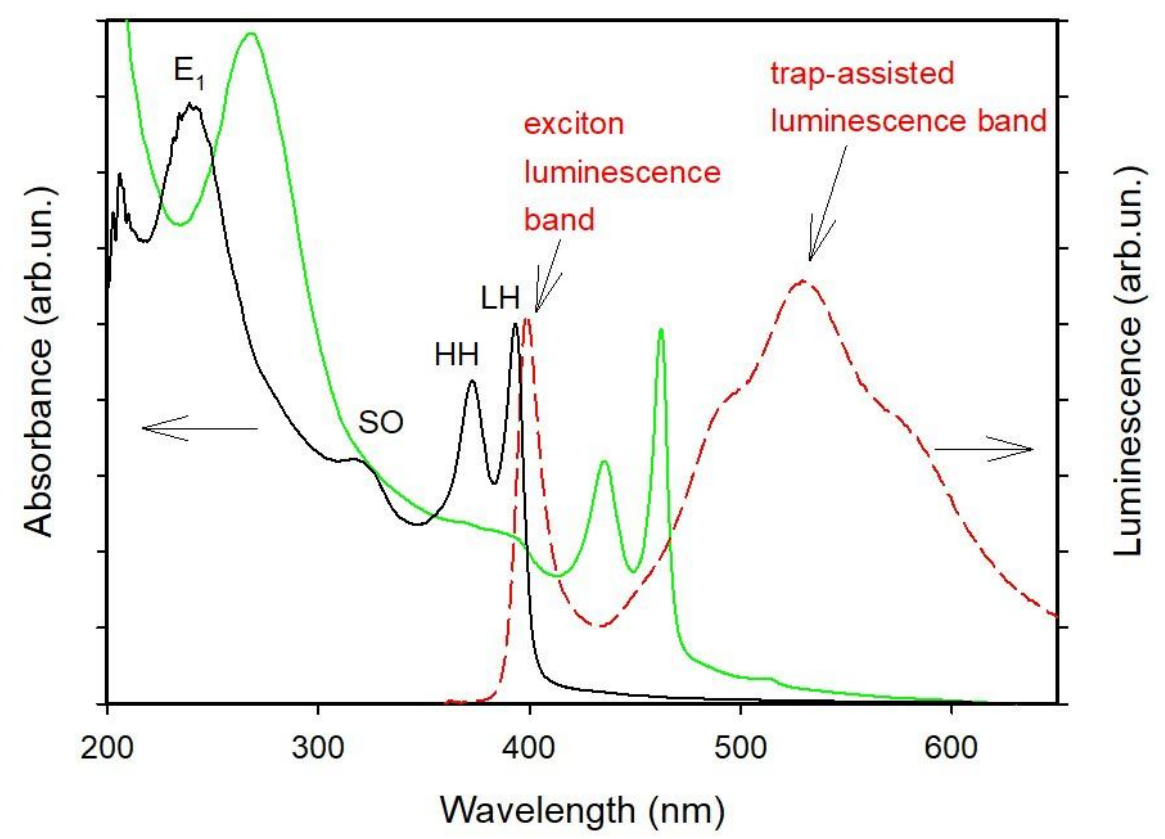

Figure S5. Typical absorption spectrum of pure CdSe394OA NSs with $150 \mathrm{~nm}$ lateral size (black lines) compared to CdSe463OA NSs (green line) in 200-700 nm spectral range. The luminescence spectrum of CdSe394OA sample with exciton at $398 \mathrm{~nm}$ and trap-assisted at 530 $\mathrm{nm}$ bands is shown by red dashed line.

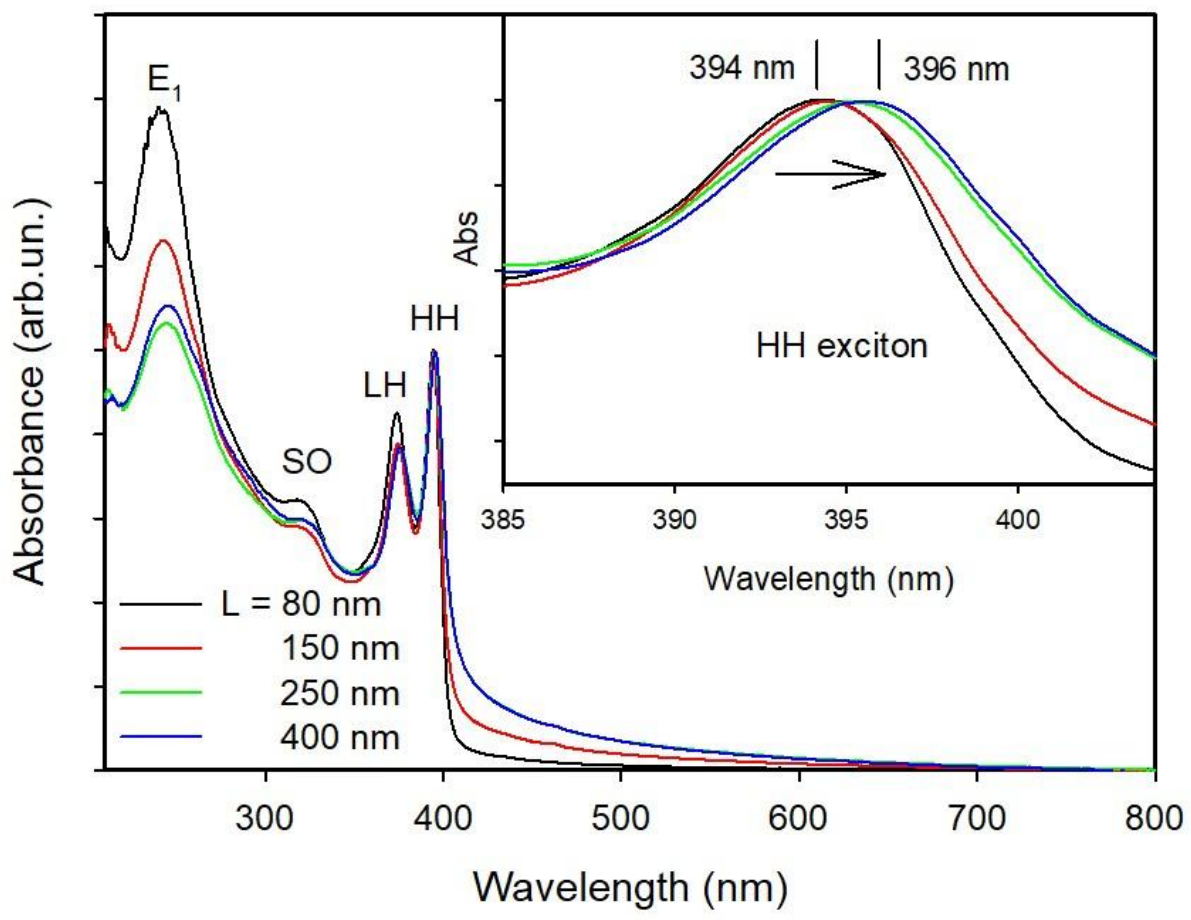

Figure S6. Modification of the absorption spectra of CdSe394OA samples with an increase in the lateral sizes of nanosheets from 80 to $400 \mathrm{~nm}$. The inset shows the enlarged region of HH exciton transition. All spectra are normalized to unity optical density at low-energy HH exciton transition. 


\section{Analysis of CdSe463/CdSAcCys samples with different lateral sizes.}
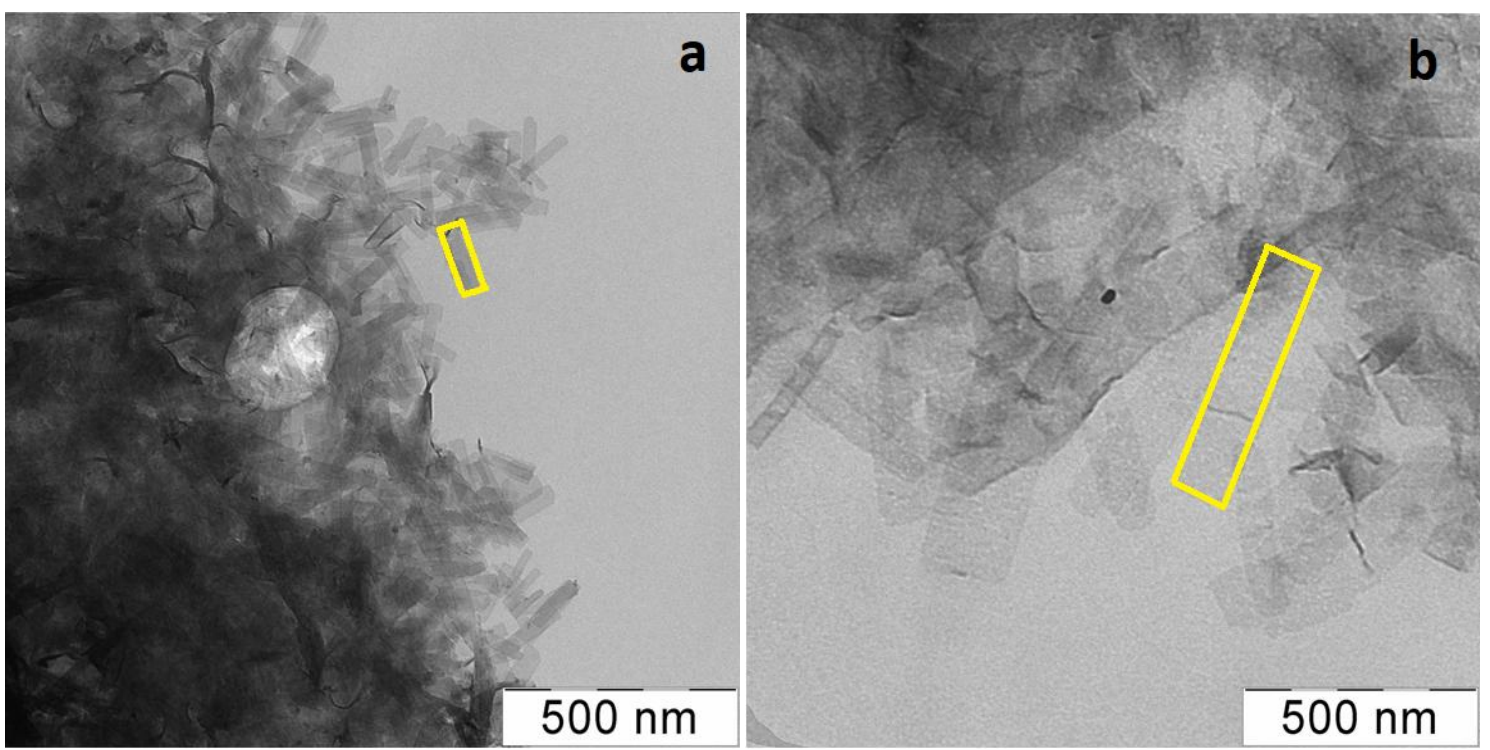

Figure S7. Overview low-resolution TEM images of CdSe463/CdSAcCys samples with lateral sizes of $110 \mathrm{~nm}(a)$ and $420 \mathrm{~nm}(\mathrm{~b})$. The yellow lines outline the contours of flat nanosheets with a rectangle shape.
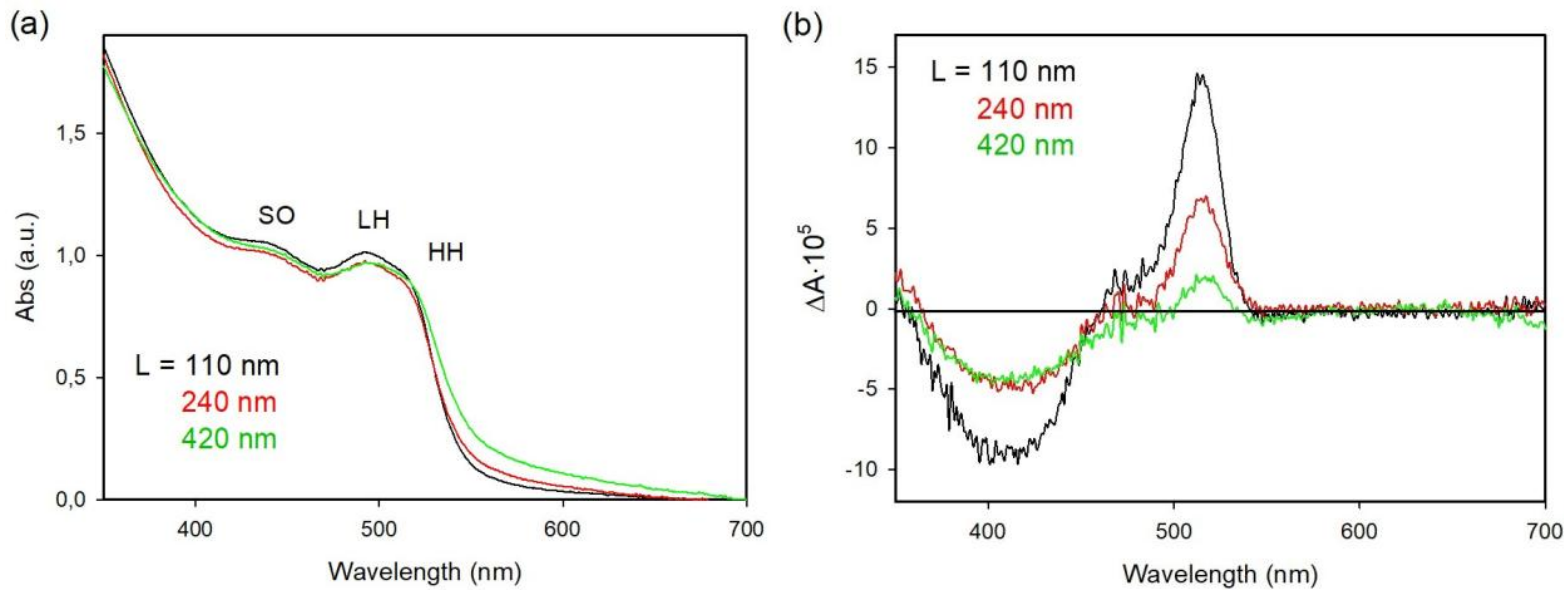

Figure S8. Absorbance (a) and CD spectra (b) of CdSe463/CdSAcCys nanosheets with lateral sizes of $110 \mathrm{~nm}$ (black line), $240 \mathrm{~nm}$ (red line), and $420 \mathrm{~nm}$ (green line), exchanged with $\mathrm{N}$ acetyl-L-cysteine . 